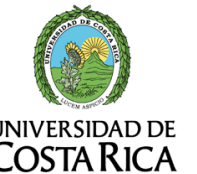

Revisión bibliográfica

Volumen 30(2):601-611. Mayo-agosto, 2019

e-ISSN 2215-3608, doi:10.15517/am.v30i2.34432

https://revistas.ucr.ac.cr/index.php/agromeso/index

\title{
Probióticos y su mecanismo de acción en alimentación animal ${ }^{1}$
}

\section{Probiotics and their mechanism of action in animal feed}

\begin{abstract}
Andrea Molina ${ }^{2}$
Recepción: 3 de setiembre, 2018. Aceptación: 14 de noviembre, 2018. Esta revisión bibliográfica formó parte de los proyectos de extensión docente ED-427 y ED-428 de la Vicerrectoría de Acción Social de la Universidad de Costa Rica. San José, Costa Rica.

2 Universidad de Costa Rica, Escuela de Zootecnia y Centro de Investigación en Nutrición Animal, 2060-501, San José, Costa Rica. andrea. molina@ucr.ac.cr (https://orcid.org/0000-0002-2852-7942).
\end{abstract}

\section{Resumen}

Introducción. En la producción pecuaria con fines comerciales, es frecuente el uso de aditivos para aumentar la efectividad de los nutrientes presentes en el alimento, su disponibilidad y absorción en el tracto gastrointestinal, además, de modular la flora intestinal de los animales y promover su crecimiento y productividad. Los antibióticos han sido de los aditivos más utilizados en las últimas décadas en producción animal en el mundo. Objetivo. El objetivo de esta revisión bibliográfica fue describir y analizar el uso de probióticos como aditivos en nutrición animal y como una alternativa al uso de antibióticos como promotores de crecimiento en la producción pecuaria comercial. Desarrollo. En esta recopilación se analiza la definición actual de probióticos aceptada por la Organización de las Naciones Unidas para la Alimentación y la Agricultura (FAO) y la Organización Mundial de la Salud (OMS), se describen los microorganismos permitidos como probióticos en nutrición animal en Costa Rica y Estados Unidos, y se explican los principales mecanismos de acción de los mismos en animales productivos. Los probióticos son organismos vivos que cuando son administrados en cantidades adecuadas confieren un beneficio para la salud del hospedero, este efecto debe estar probado y superar al efecto dado por el placebo. Los microorganismos usados como probióticos en nutrición animal no deben ser patógenos para los animales y deben ser resistentes a los procesos de elaboración de alimentos y piensos. Se ha reportado que el beneficio de los probióticos en animales productivos, se debe principalmente a que estos fomentan el balance microbiano en el tubo digestivo y la modulación del sistema inmune, resultando en un aumento en la digestión y absorción de nutrientes, y disminuyen la incidencia de enfermedades infecciosas. Conclusión. El uso de diferentes probióticos en animales productivos para aumentar el rendimiento y la salud animal, ha sido comprobado en diferentes especies productivas y su uso es promisorio como promotor de crecimiento en lugar de antibióticos.

Palabras clave: microorganismos benéficos, salud animal, piensos, aditivos, promotores del crecimiento, flora microbiana.

\begin{abstract}
Introduction. In the livestock production for commercial purposes, additives are frequently used to increase the effectiveness of nutrients present in the food, its availability, and absorption in the gastrointestinal tract, as well as modulate the intestinal flora of animals and promote their growth and productivity. Antibiotics have been among the
\end{abstract}


most used additives in recent decades animal production worldwide. Objective. The aim of this literature review was to describe the use of probiotics as additives in animal nutrition, as an alternative to the use of antibiotics as growth promoters in commercial livestock production. Development. This compilation analyzes the current definition of probiotics accepted by the Food and Agriculture Organization of the United Nations (FAO) and the World Health Organization (WHO) it also describres the microorganisms allowed as probiotics in animal nutrition in Costa Rica and the United States. Additionally,the main mechanisms of action in productive animals are explained. Probiotics are living organisms that when administered in adequate amounts confer a benefit to the health of the host, this effect must be proven and overcome the effect given by the placebo. The microorganisms used as probiotics in animal nutrition should not be pathogenic for animals, and should be resistant to the processes of food and feed elaboration. It has been reported that the benefit of probiotics in productive animals is mainly due to the fact that these promote the microbial balance in the digestive tract and the modulation of the immune system, resulting in an increase in the digestion and absorption of nutrients and decreasing the incidence of infectious diseases. Conclusion. The use of different probiotics in productive animals to increase productivity and animal health, has been proven in different commercial livestock species and its use is promising as a growth promoter instead of antibiotics.

Keywords: beneficial microorganisms, animal health, foods, feed additive, growth promoters, microbial flora.

\section{Introducción}

En la producción pecuaria con fines comerciales, es frecuente el uso de aditivos para aumentar la efectividad de los nutrientes presentes en el alimento, su disponibilidad y absorción en el tubo digestivo, además de modular la flora intestinal de los animales y promover su crecimiento. El nivel de aditivos que normalmente deben ser adicionados a los alimentos para animales, en función de aumentar el crecimiento y una mayor productividad, es bajo. Este aumento en la productividad y crecimiento, disminuye los costos de los productos de origen animal (Chahal et al., 2008). Entre los productos usados como aditivos en alimentos para animales, están antibióticos, probióticos, oligosacáridos, enzimas, ácidos orgánicos, vitaminas y minerales, entre otros. El uso de aditivos se asocia con un producto final más homogéneo y de mayor calidad (Yirga, 2015). Los antibióticos han sido de los aditivos más utilizados en las últimas décadas, esto debido a su efecto terapéutico que ha servido para disminuir infecciones como la diarrea, resultando en una mejora en parámetros productivos como ganancia de peso y conversión alimenticia. Los efectos reportados de los antibióticos como aditivos en alimentos para animales, se deben principalmente a la modificación de la población bacteriana intestinal, su interacción con la pared intestinal y con el sistema inmune del animal (Diarra y Malouin, 2014).

El uso de antibióticos como promotores del crecimiento en la producción pecuaria, se ha asociado con un aumento en la aparición de bacterias resistentes a los antibióticos, ya que se usan en dosis menores a las necesarias para eliminar toda la población bacteriana y dejan algunas bacterias que logran resistir. Estas logran reproducirse, lo que resulta en la multiplicación de genes de resistencia y diseminación mediante transferencia horizontal hacia diferentes especies bacterianas, tanto patógenas como comensales, dentro del tubo digestivo del animal (Dutil et al., 2010; Carattoli, 2013; Yirga, 2015). La unión europea ha prohibido el uso de antibióticos como aditivos en alimentos para animales desde el 2006 (Yirga, 2015). La Agencia de Administración de Alimentos y Medicamentos (FDA, por sus siglas en inglés) de Estados Unidos, prohibió a partir de enero del 2017 el uso de antibióticos (exceptuando coccidiostatos) como promotores de crecimiento en alimentación animal.

Esto ha fomentado la búsqueda de otros productos alternativos para usarlos como promotores del crecimiento y de la salud en animales productivos. Entre las alternativas que se han venido utilizando en alimentación animal, están 
los prebióticos, probióticos (Uyeno et al., 2015), ácidos orgánicos, aceites esenciales (Gadde et al., 2017; GranadosChinchilla, 2017), entre otros. El objetivo de esta revisión bibliográfica fue describir y analizar el uso de probióticos como aditivos en nutrición animal, y como una alternativa al uso de antibióticos como promotores de crecimiento en la producción pecuaria comercial, así como los mecanismos de acción de estos destinados a la producción de alimentos.

\section{Probióticos: definición, legislación}

Los probióticos han sido definidos por la Organización de las Naciones Unidas para la Alimentación y la Agricultura (FAO) y la Organización Mundial de la Salud (OMS), como organismos vivos que cuando se administran en cantidades adecuadas confieren un beneficio para la salud del hospedero (FAO/WHO, 2001). Cabe resaltar que, como lo indica esta definición, el término probiótico no está limitado a alimentos o a una administración oral del producto, este término puede utilizarse también en productos de aplicación cutánea, intravenosa, entre otras. Los probióticos son organismos vivos y no está limitado el tipo de organismo, pueden ser bacterias, hongos, entre otros (FAO/WHO, 2001; Reid, 2016). Como lo aclara la definición de la OMS y la FAO, los microorganismos empleados deben estar vivos para que puedan ser clasificados como probióticos. No existen probióticos muertos, esto es un mal uso del término. Otro aspecto importante a la hora de clasificar un producto como probióticos es que debe existir un efecto beneficioso en el animal o persona a la que se le administra, y este efecto debe estar probado y debe superar al efecto dado por el placebo (FAO/WHO, 2002; Hill et al., 2014).

Entre los productos comerciales utilizados actualmente como probióticos en la alimentación animal, se encuentra una amplia variedad de presentaciones; algunos emplean una sola especie microbiana, otros son multiespecie, además, en el mercado se encuentran probióticos a base de bacterias, hongos, microorganismos formadores y no formadores de esporas (Bajagai et al., 2016). Entre esta variedad de productos están los probióticos autóctonos, que son los que utilizan microorganismos que son parte de la flora indígena del tracto gastrointestinal de los animales, como las bacterias pertenecientes a los géneros Lactobacillus y Bifidobacterium, y los probióticos alóctonos que usan microorganismos que normalmente no están presentes dentro del tubo digestivo de los animales, como es el caso de las levaduras (Bajagai et al., 2016).

Los microorganismos deben reunir ciertas características para ser usados como probióticos en nutrición animal. No deben ser patógenos para los animales, deben ser resistentes a factores físicos y ambientales propios de los procesos de elaboración de alimentos para animales, a saber: calor, desecación, radiación UV. Deben manter su viabilidad durante el procesamiento, almacenamiento y manejo (Setlow, 2006; Cutting, 2011), además de ser capaces de resistir el ambiente del tubo digestivo, adherirse a la pared intestinal y colonizar el tubo digestivo del animal. Estos microorganismos deben tener la capacidad de crecer rápidamente en medios de cultivo de bajo costo, para que su producción y uso en nutrición animal sea rentable (Bajagai et al., 2016). Debido a esto, el uso de bacterias formadoras de esporas altamente resistentes a condiciones ambientales adversas como altas temperaturas y desecación, particularmente pertenecientes al género Bacillus, son cada vez más frecuentes (Reid, 2016).

Actualmente existen muchos productos en el mercado local e internacional que usan la denominación de probióticos, sin embargo, según FAO/WHO (2002) y Hill et al. (2014), no han pasado por pruebas que logren comprobar su efecto benéfico en nutrición animal. Por ejemplo, en Canadá existe una guía para los consumidores donde aparecen los probióticos registrados en el mercado canadiense para administración en humanos y que han sido probados de forma satisfactoria. En dicha guía se hace referencia a los artículos científicos que respaldan las pruebas y exactamente para qué patología ha sido probado cada probiótico (Skokovic-Sunjic, 2016). Antes de usar probióticos comerciales en producción animal, es importante revisar los respaldos científicos con que cuenta el producto y si la especie, edad del animal y efecto reportado, concuerdan con las necesidades de la producción pecuaria a la que se van a proporcionar. 
En cuanto a la regulación en la comercialización de probióticos y del uso de esa denominación en las etiquetas nutricionales, existen amplias diferencias entre países y regiones. En la Unión Europea la autoridad en seguridad alimentaria (EFSA), prácticamente ha limitado el uso del término probiótico en las etiquetas, ya que para ellos son pocos los productos registrados que han presentado pruebas sobre su efecto y eficacia por encima del efecto del placebo (Yeoman y White, 2014).

En la Cuadro 1 se presentan los microorganismos vivos permitidos en alimentos para animales en Costa Rica y en los estados miembros de la Asociación Americana de Oficiales de Control de Alimentos para Animales (Jarman, 2018).

Cuadro 1. Microorganismos permitidos en alimentos para animales por la Asociación Americana de Oficiales de Control de Alimentos para Animales. Modificado de la publicación Jarman (2018).

Table 1. Microorganisms allowed in animal feed by the Association of American Feed Control Officials. Modified from the publication Jarman (2018).

\begin{tabular}{|c|c|}
\hline \multicolumn{2}{|c|}{ Microorganismos } \\
\hline Hongo filamentoso & Bacterias Gram +, no formadora de espora \\
\hline Aspergillus Niger & Bifidobacterium adolescentis \\
\hline Aspergillus orizae & Bifidobacterium animalis \\
\hline Levadura & Bifidobacterium bifidum \\
\hline Saccharomyces cerevisiae & Bifidobacterium infantis \\
\hline Bacterias Gram +, formadora de esporas & Bifidobacterium longum \\
\hline Bacillus coagulants & Bifidobacterium thermophilum \\
\hline Bacillus lentus & Enterococcus cremoris \\
\hline Bacillus licheniformis & Enterococcus diacetylactis \\
\hline Bacillus pumilus & Enterococcus faecium \\
\hline Bacillus subtilis & Enterococcus intermedius \\
\hline Bacterias Gram - , no formadora de espora & Enterococcus lactis \\
\hline Bacteriodes amylophilus & Enterococcus thermophilus \\
\hline Bacteriodes capillosus & Lactobacillus acidolphilus \\
\hline Bacteriodes ruminocola & Lactobacillus brevis \\
\hline Bacteriodes suis & Lactobacillus buchneri (solo en ganado) \\
\hline Megasphaera elsdenii (solo ganado) & Lactobacillus bulgaricus \\
\hline & Lactobacillus casei \\
\hline & Lactobacillus cellobiosus \\
\hline & Lactobacillus curvatus \\
\hline & Lactobacillus delbruekii \\
\hline & Lactobacillus farciminis (solo en cerdos) \\
\hline & Lactobacillus fermentum \\
\hline & Lactobacillus helveticus \\
\hline & Lactobacillus lactis \\
\hline & Lactobacillus plantarum \\
\hline & Lactobacillus reuterii \\
\hline & Leuconostoc mesenteroides \\
\hline & Pediococcus acidilacticii \\
\hline & Pediococcus cerevisiae (damnosus) \\
\hline & Pediococcus pentosaceus \\
\hline & Propionibacterium acidipropionici (solo ganado) \\
\hline & Propionibacterium freudenreichii \\
\hline & Propionibacterium shermanii \\
\hline
\end{tabular}




\section{Mecanismos de acción de los probióticos en animales}

Las comunidades o poblaciones microbianas en el sistema digestivo de animales domésticos, son muy complejas y pueden estar formadas por bacterias, protozoarios, hongos y virus. Estos microorganismos llevan a cabo procesos de digestión y fermentación de polímeros vegetales, síntesis de vitaminas, bioconversión de compuestos tóxicos, estimulación del sistema inmune, mantenimiento de la peristalsis intestinal, mantenimiento de la integridad de la mucosa intestinal y sirven como barrera contra la colonización por patógenos (ChaucheyrasDurand y Durand, 2010). Estos mismos autores sugieren que la microbiota afecta directamente la eficacia de la alimentación, productividad, salud y bienestar de los animales. La microbiota del tubo digestivo de animales productivos, puede variar dependiendo de las prácticas de alimentación, de la composición de las dietas y del manejo en finca, entre otros.

En rumiantes, la microbiota ruminal es responsable de proporcionar aproximadamente el $70 \%$ de los requerimientos diarios de energía (Flint y Bayer, 2008; Yeoman y White, 2014). Los microorganismos que forman parte de la microbiota del rumen, colectivamente poseen diversas enzimas con la capacidad de hidrolizar carbohidratos estructurales como celulosa, xilanos, mananos, pectinas, inulina, beta glucanos y almidones resistentes (que no son digeribles por los animales) (Hess et al., 2011; Yeoman y White, 2014), y producir ácidos grasos de cadena corta como acetato, propionato y butirato, los cuales tienen un papel muy importante en la salud y nutrición animal (Hess et al., 2011; Yeoman y White, 2014).

Se ha reportado que el beneficio de los probióticos en la salud de los animales, se debe principalmente a que estos fomentan el balance microbiano en el tubo digestivo. Los mecanismos por los cuales los probióticos logran este balance son exclusión competitiva, antagonismo bacteriano e inmuno-modulación (Bajagai et al., 2016; Markowiak y Śliżewska, 2018).

\section{Modificación de la microbiota y ambiente del tubo digestivo}

Uno de los mecanismos de los probióticos consiste en cambiar la dinámica de la población microbiana, disminuir el crecimiento de microorganismos patógenos y promover el crecimiento de microflora beneficiosa (An et al., 2008; Mountzouris et al., 2009). Poblaciones microbianas benéficas en el tubo digestivo, se han asociado con aumento en el rendimiento del animal, lo que refleja una digestión más eficiente y mejora en la inmunidad (Niba et al., 2009; Hung et al., 2012).

La capacidad de los probióticos de reducir los microorganismos patógenos en el tubo digestivo, puede ser deberse a la producción de bacteriocinas (Corr et al., 2007; Shim et al., 2012), a la exclusión por competencia al adherirse los probióticos al epitelio intestinal, al cambio en $\mathrm{pH}$ y a la inducción de la respuesta del sistema inmune (Jordan et al., 2014).

En aves que recibieron probióticos como aditivos en su dieta, se reportaron aumentos de la población de Lactobacillus y Bifidobacteria, disminución en la población de Escherichia coli (Mookiah et al., 2014; Zhang y Kim, 2014) y disminución en la población de Clostridium spp. (Shim et al., 2012; Liu et al., 2014; Zhang et al., 2016). La bacterias L. reuteri, B. subtillis y B. licheniformis, han demostrado en lechones la capacidad de disminuir la cantidad de E. coli y Salmonella sp. en las heces (Ahmed et al., 2014). Esta disminución en la excreción de microorganismos patógenos, reduce el riesgo de infección de otros animales en las producciones pecuarias y la contaminación cruzada. La modificación en la población del tubo digestivo, se ha visto con las bacterias comúnmente usadas como probióticos tanto gram negativas como gram positivas, bacterias ácido lácticas y bacterias formadoras de esporas como Bacillus sp. (Shim et al., 2012; Abdelqader et al., 2013). 
Algunas de las especies bacterianas comúnmente usadas como probióticos, entre ellas bacterias ácido lácticas como Bifidobacterium, Bacillus y Lactobacillus, producen sustancias (bacteriocinas) que tienen la capacidad de inhibir el crecimiento de microorganismos patógenos como Staphylococcus, Enterococcus, Listeria y Salmonella en el intestino de los animales (Cheikhyoussef et al., 2008; Surendran et al., 2017). Las bacteriocinas inhiben la síntesis de la pared celular bacteriana, produciéndose poros en la superficie bacteriana (Hassan et al., 2012).

Los géneros Lactobacillus y Bifidobacterium producen ácidos orgánicos como ácido láctico y acético, que pueden ser tomados por otras bacterias, entre ellas patógenas, en el tubo digestivo, reducen su pH intracelular a niveles letales (Daskiran et al., 2012). Estos ácidos orgánicos, además pueden contribuir a la disminución del pH en el tubo digestivo, creando condiciones favorables para la microbiota existente y disminuyendo el riesgo de ser colonizado por microorganismos patógenos (Chaucheyras-Durand y Durand, 2010).

Los microorganismos suministrados como probióticos podrían colonizar el tubo digestivo de animales jóvenes y adultos, previniendo la colonización por microorganismos patógenos. Algunas cepas de Lactobacillus y Bifidobacterium tienen proteínas de superficie hidrofóbica que favorecen la adhesión inespecífica a células animales, cubren los sitios de unión del receptor y evitan la unión del epitelio intestinal con microorganismos patógenos (Johnson-Henry et al., 2007; Konstantinov et al., 2008). Se ha reportado la adhesión de Lactobacillus sp. al epitelio intestinal en pollos de engorde, lo que podría excluir por competencia a microorganismos patógenos (Mookiah et al., 2014).

Uno de los posibles mecanismos para reforzar la integridad de la barrera intestinal y evitar el ingreso de patógenos y toxinas al animal, es aumentar la expresión de genes involucrados en la transmisión de señales, entre las uniones estrechas "tight junction" de las células del epitelio intestinal (Bajagai et al., 2016). Se ha reportado en algunos probióticos como Lactobacillus, la modulación de genes que codifican por proteínas de adherencia dentro de estas uniones estrechas como E-cadherin y E-catenin (Anderson et al., 2010; Bermudez-Brito et al., 2012).

Algunas bacterias usadas como probióticos tienen la capacidad de reducir la permeabilidad del epitelio intestinal, disminuyen la translocación de patógenos intestinales del tubo digestivo hacia otros órganos como el hígado, el bazo y nódulos linfáticos en ratones (Llopis et al., 2005) y cerdos (Dowarah et al., 2016). Para que los probióticos puedan ayudar de forma efectiva a mantener la función de barrera de la pared intestinal, es crucial la administración del probióticos antes de que los patógenos se hayan multiplicado en el tubo digestivo. Este efecto es dependiente de la especie usada como probiótico y del tipo de patógeno (virus, bacteria u hongo) (Lodemann, 2010).

Se ha reportado que algunas bacterias usadas como probióticos, secretan señales químicas llamadas auto inductores que afectan el comportamiento de otras bacterias y hasta del hospedero. Este proceso de comunicación bacteriana recibe el nombre de quorum sensing. Estas señales enviadas por bacterias probióticas podrían tener un efecto en la virulencia de bacterias patógenas afectando, por ejemplo la producción de toxinas (Medellin-Peña et al., 2007).

\section{Modulación del sistema inmune}

Se ha descrito que los probióticos tienen la capacidad de modular la respuesta inmune del hospedero, tanto la respuesta innata como la respuesta adaptativa (Patel et al., 2015; Smolinska et al., 2017).

Varios estudios han demostrado los efectos inmunoestimuladores de los probióticos, Bai et al. (2013), indicaron que un probiótico que contenía L. fermentum y S. cerevisiae, estimuló la respuesta de células $\mathrm{T}$ intestinales, caracterizada por una mayor producción de linfocitos T CD3 +, CD4 + y CD8 + en el tubo digestivo de pollos de engorde. También se ha reportado que los probióticos aumentan el nivel de inmunoglobulinas en el suero (Zhang y Kim, 2014), y el título de anticuerpos contra la enfermedad de Newcastle en pollos (Landy y Kavyani, 2013). En cerdos L. reuteri, B. subtillis y B. licheniformis, aumentaron los niveles en suero de Inmunoglobulina G (Ahmed et al., 2014). En contraposición, Siepert et al. (2014) reportaron un efecto inmunosupresor del probiótico E. faecium 
NCIMB 10415 en lechones, que redujo la proliferación de células mononucleares sanguíneas y la expresión de los genes asociados a inmunidad intestinal, IL-8, IL-10 y CD86, en respuesta a Salmonella Typhimurium. También se ha visto disminución en la expresión de la interleucina b (IL-b) en el ileum de lechones (Liu et al., 2014).

\section{Aumento en la digestión y absorción de nutrientes}

El uso de probióticos como promotores de crecimiento en alimentación animal se debe principalmente a la mejora reportada en producción, asociada con un aumento en la digestión y absorción de nutrientes (Bajagai et al., 2016). En pollos de engorde que recibieron L. bulgaricus en la dieta, se observó un aumento significativo en proteína y grasa, una ganancia significativa de peso (Apata, 2008; Zhang y Kim, 2014), y aumento en la disponibilidad del calcio (Chawla et al., 2013). Por otro lado, Li et al. (2008) no observaron una variación significativa en ganancia de peso, consumo de alimento y conversión alimenticia en pollos de engorde que recibieron un probiótico comercial en su dieta. En cerdos que recibieron probiótico a base de diferentes especies de Lactobacillus, hubo un aumento significativo en la ganancia de peso diaria, reducción en la incidencia de diarrea en comparación con el grupo control (Liu et al., 2014), y aumento en la digestibilidad de nutrientes (Ahmed et al., 2014).

La mayor digestibilidad de nutrientes podría deberse al aumento de la actividad enzimática en el intestino, ocasionada por los probióticos administrados. Investigaciones han reportado un aumento en la actividad enzimática de la amilasa, al agregar Lactobacillus a la dieta de pollos de engorde (Jin et al., 2000), y aumento en la actividad de la sucrosa y la lactasa al agregar Lactobacillus en la dieta de cerdos (Collington et al., 1990; Bajagai et al., 2016). Bacillus amyloliquefaciens, la cual se ha utilizado como probiótico en alimentación animal, produce enzimas extracelulares como amilasa, celulasa, proteasas y metaloproteasas, lo que podría favorecer la digestión de nutrientes (Lee et al., 2008). También se ha descrito que el consumo de probióticos aumenta la altura de las vellosidades intestinales en pollos, aumentando el área de absorción de nutrientes (Afsharmanesh y Sadaghi, 2014).

\section{Conclusiones}

El uso de probióticos en animales productivos para aumentar el rendimiento y la salud animal, ha sido comprobado en diferentes especies productivas y su uso como promotor de crecimiento en lugar de antibióticos es promisoria. Los efectos que se han observado en animales, dependen de muchos factores entre ellos: las especies microbianas empleadas, la especie animal en la cual se desea aumentar la productividad, la edad de los animales y la condición de la flora del tubo digestivo existente en los animales antes de iniciar la administración de probióticos.

\section{Literatura citada}

Abdelqader, A., R. Irshaid, and A.R. Al-Fataftah. 2013. Effects of dietary probiotic inclusion on performance, eggshell quality, cecal microflora composition, and tibia traits of laying hens in the late phase of production. Trop. Anim. Health. Prod. 45:1017-1024. doi:10.1007/s11250-012-0326-7

Afsharmanesh, M., and B. Sadaghi. 2014. Effects of dietary alternatives (probiotic, green tea powder and Kombucha tea) as antimicrobial growth promoters on growth, ileal nutrient digestibility, blood parameters, and immune response of broiler chickens. Comp. Clin. Pathol. 23:717-724. doi:10.1007/s00580-013-1676-x 
Ahmed, S.T., J. Hoon, H.S. Mun, and C.J. Yang. 2014. Evaluation of Lactobacillus and Bacillus-based probiotics as alternatives to antibiotics in enteric microbial challenged weaned piglets. Afr. J. Microbiol. Res. 8:96-104. doi:10.5897/ AJMR2013.6355

An, B., B. Cho, S. You, H. Paik, H. Chang, S. Kim, C. Yun, and C. Kang. 2008. Growth per-formance and antibody response of broiler chicks fed yeast derived $\beta$-glucan and single-strain probiotics. Asian-Australas. J. Anim. Sci. 21:1027-1032. doi:10.5713/ajas.2008.70571

Anderson, R.C., A.L. Cookson, W.C. McNabb, Z. Park, M.J. McCann, W.J. Kelly, and N.C. Roy. 2010. Lactobacillus plantarum MB452 enhances the function of the intestinal barrier by increasing the expression levels of genes involved in tight junction formation. BMC Microbiol. 10:316. doi:10.1186/1471-2180-10-316

Apata, D. 2008. Growth performance, nutrient digestibility and immune response of broiler chicks fed diets supplemented with a culture of Lactobacillus bulgaricus. J. Sci. Food Agric. 88:1253-1258. doi:10.1002/jsfa.3214

Bai, S., A. Wu, X. Ding, Y. Lei, J. Bai, K. Zhang, and J. Chio. 2013. Effects of probiotic-sup-plemented diets on growth performance and intestinal immune characteristics of broiler chickens. Poult. Sci. 92:663-670. doi:10.3382/ps.201202813.

Bajagai, Y.S., A.V. Klieve, P.J. Dart, and W.L. Bryden. 2016. Probiotics in animal nutrition: Production, impact and regulation. paper 179. FAO Animal Production and Health, Rome, ITA.

Bermudez-Brito, M., J. Plaza-Díaz, S. Muñoz-Quezada, C. Gómez-Llorente, and A. Gil. 2012. Probiotic mechanisms of action. Ann. Nutr. Metab. 61:160-174. doi:10.1159/000342079

Carattoli, A. 2013. Plasmids and the spread of resistance. Int. J. Med. Microbiol. 303(6-7):298-304. doi:10.1016/j. ijmm.2013.02.001.

Chahal, U.S., P.S. Niranjan, and S. Kumar. 2008. Handbook of general animal nutrition. International Book Distributing Co., IND.

Chaucheyras-Durand, F., and H.M. Durand. 2010. Probiotics in animal nutrition and health. Benef. Microbes 11:3-9. doi:10.3920/BM2008

Chawla, S., S. Katoch, K. Sharma, and V. Sharma. 2013. Biological response of broiler supple-mented with varying dose of direct fed microbial. Vet. World 6:521-524. doi:10.5455/vetworld.2013.521-524

Cheikhyoussef,A.,N.Pogori, W.Chen, and H.Zhang. 2008.Antimicrobial proteinaceous compounds obtained from bifidobacteria: From production to their application. Int. J. Food Microbiol. 125:215-222. doi:10.1016/j.ijfoodmicro.2008.03.012.

Collington, G., D. Parker, and D. Armstrong. 1990. The influence of inclusion of either an antibiotic or a probiotic in the diet on the development of digestive enzyme activity in the pig. Br. J. Nutr. 64:59-70. doi:10.1079/BJN19900009

Corr, S.C., Y. Li, C.U. Riedel, P.W. O'Toole, C. Hill, and C.G. Gahan. 2007. Bacteriocin production as a mechanism for the antiinfective activity of Lactobacillus salivarius UCC118. PNAS 104:7617-7621. doi:10.1073/pnas.0700440104

Cutting, S.M. 2011. Bacillus probiotics. Food Microbiol. 28:214-220. doi:10.1016/j.fm.2010.03.007

Daskiran, M., A.G. Onol, O. Cengiz, H. Unsal, S. Turkyilmaz, O. Tatli, and O. Sevim. 2012. Influence of dietary probiotic inclusion on growth performance, blood parameters, and intestinal microflora of male broiler chickens exposed to posthatch holding time. J. Appl. Poult. Res. 21:612-622. doi:10.3382/japr.2011-00512

Diarra, M.S., and F. Malouin. 2014. Antibiotics in Canadian poultry productions and anticipated alternatives. Front. Microbiol. 5:282. doi:10.3389/fmicb.2014.00282 
Dowarah, R., A.K. Verma, and N. Agarwal. 2016. The use of Lactobacillus as an alternative of antibiotic growth promoters in pigs: a review. Anim. Nutr. 3:1-6. doi:10.1016/j.aninu.2016.11.002

Dutil, L., R. Irwin, R. Finley, L.K. Ng, B. Avery, P. Boerlin, and D.R. Pillai. 2010. Ceftiofur resistance in Salmonella enterica serovar Heidelberg from chicken meat and humans, Canada. Emerg. Infect. Dis. 16:48-54. doi:10.3201/eid1601.090729

FAO/WHO. 2001. Evaluation of health and nutritional properties of powder milk and live lactic acid bacteria. FAO/WHO, ARG.

FAO/WHO. 2002. Guidelines for the evaluation of probiotics in food. FAO/WHO, CAN.

Flint, H.J., and E.A. Bayer. 2008. Plant cell wall breakdown by anaerobic microorganisms from the mammalian digestive tract. Ann. N. Y. Acad. Sci. 1125:280-288. doi:10.1196/annals.1419.022

Gadde, U., W. Kim, S. Oh, and H. Lillehoj. 2017. Alternatives to antibiotics for maximizing growth performance and feed efficiency in poultry: A review. Anim. Health Res. Rev. 18:26-45. doi:10.1017/S1466252316000207

Granados-Chinchilla, F. 2017. A review on phytochemicals (Including essential oils and extracts) inclusion in feed and their effects on food producing animals. J. Vet. Med. Sci. 3:555620. doi:10.19080/JDVS.2017.03.555620002

Hassan, M., M. Kjos, I. Nes, D. Diep, and F. Lotfipour. 2012. Natural antimicrobial peptides from bacteria: characteristics and potential applications to fight against antibiotic resistance. J. App. Microbiol. 113:723-736. doi:10.1111 /j.1365-2672.2012.05338

Hess, M., A. Sczyrba, R. Egan, T.W. Kim, H. Chokhawala, G. Schroth, S. Luo, D.S. Clark, F. Chen, T. Zhang, R.I. Mackie, L.A. Pennacchio, S.G. Tringe, A. Visel, T. Woyke, Z. Wang, and E.M. Rubin. 2011. Metagenomic discovery of biomass degrading genes and genomes from cow rumen. Science 331:463-467. doi:10.1126/science.1200387

Hill, C., F. Guarner, G. Reid, G.R. Gibson, D.J. Merenstein, B. Pot, M. Morelli, R.B. Canani, H.J. Flint, S. Salminen, P.C. Calder, and M.E. Sanders. 2014. The international scientific association for probiotics and prebiotics consensus statement on the scope and appropriate use of the term probiotic. Nat. Rev. Gastroenterol. Hepatol. 1:506-514. doi:10.1038/ nrgastro.2014.66

Hung, A.T., S.T. Lin, T.Y. Yang, C.K. Chou, H.C. Liu, J.J. Lu, B. Wang, S.Y. Chen, and T.F. Lien. 2012. Effects of Bacillus coagulans ATCC 7050 on growth performance, intestinal morphology, and microflora composition in broiler chickens. Anim. Prod. Sci. 52:874-879. doi:10.1071/AN11332

Jarman, J. 2018. Fermentation products. In: AAFCO, editor, AAFCO 2018 Official publication. AAFCO, Atlanta, GA, USA. p. 384-388.

Jin, L., Y. Ho, N. Abdullah, and S. Jalaludin. 2000. Digestive and bacterial enzyme activities in broilers fed diets supplemented with Lactobacillus cultures. Poult. Sci. 79:886-891. doi:10.1093/ps/79.6.886

Johnson-Henry, K.C., K.E. Hagen, M. Gordonpour, T.A. Tompkins, and P.M. Sherman. 2007. Surface-layer protein extracts from Lactobacillus helveticus inhibit enterohaemorrhagic Escherichia coli O157: H7 adhesion to epithelial cells. Cell. Microbiol. 9:356-367. doi:10.1111/j.1462-5822.2006.00791.x

Jordan, K., M. Dalmasso, J. Zentek, A. Mader, G. Bruggeman, J. Wallace, D. De Medici, A. Fiore, E. Prukner-Radovcic, M. Lukac. L. Axelsson, A. Holck, H. Ingmer, and M. Malakauskas. 2014. Microbes versus microbes: control of pathogens in the food chain. J. Sci. Food. Agric. 94:3079-3089. doi:10.1002/jsfa.6735

Konstantinov, S.R., H. Smidt, A.D. Akkermans, L. Casini, P. Trevisi, M. Mazzoni, S. De-Filippi, P. Bosi, and W. de-Vos. 2008. Feeding of Lactobacillus sobrius reduces Escherichia coli F4 levels in the gut and promotes growth of infected piglets. FEMS Microbiol. Ecol. 66:599-607. doi:10.1111/j.1574-6941.2008.00517.x

Landy, N., and A. Kavyani. 2013. Effects of using a multi-strain probiotic on performance, immune responses and caecal microflora composition in broiler chickens reared under cyclic heat stress condition. Iran. J. Appl. Anim. Sci. 3:703-708. 
Lee, Y.J., B.K. Kim, B.H. Lee, K.I. Jo, N.K. Lee, C.H. Chung, Y.C. Lee, and J.W. Lee. 2008. Purification and characterization of cellulase produced by Bacillus amyoliquefaciens DL-3 utilizing rice hull. Biores. Technol. 99:378-386. doi:10.1016/j. biortech.2006.12.013.

Li, L.L., Z.P. Hou, T.J. Li, G.Y. Wu, R.L. Huang, Z.R. Tang, C.B. Yang, J. Gong, H. Yu, and X.F. Kong. 2008. Effects of dietary probiotic supplementation on ileal digestibility of nutrients and growth performance in 1- to 42-day-old broilers. J. Sci. Food. Agric. 88:35-42. doi:10.1002/jsfa.2910

Liu, H., J. Zhang, S.H. Zhang, F.J. Yang, P.A. Thacker, G.L. Zhang, S. Qiao, and X. Ma. 2014. Oral administration of Lactobacillus fermentum 15007 favors intestinal development and alters the intestinal microbiota in formula-fed piglets. J. Agric. Food. Chem. 62:860-866. doi:10.1021/jf403288r

Llopis, M., M. Antolin, F. Guarner, A. Salas, and J. Malagelada. 2005. Mucosal colonisation with Lactobacillus casei mitigates barrier injury induced by exposure to trinitronbenzene sulphonic acid. Gut. 54:955-959. doi:10.1136/gut.2004.056101

Lodemann, U. 2010. Effects of probiotics on intestinal transport and epithelial barrier function. In: R. Ross, and V. Preedy, editors, Bioactive foods in promoting health: probiotics and prebiotics. Academic Press, Waltham, MA, USA. p. 303-333.

Markowiak, P., and K. Śliżewska. 2018. The role of probiotics, prebiotics and synbiotics in animal nutrition. Gut Pathog. 10:21. doi:10.1186/s13099-018-0250-0

Medellin-Peña, M.J., H. Wang, R. Johnson, S. Anand, and M.W. Griffiths. 2007. Probiotics affect virulence-related gene expression in Escherichia coli O157: H7. App. Environ. Microbiol. 73:4259-4267. doi:10.1128/AEM.00159-07

Mountzouris, K.C., C. Balaskas, I. Xanthakos, A. Tzivinikou, and K. Fegeros. 2009. Effects of a multi-species probiotic on biomarkers of competitive exclusion efficacy in broilers chal-lenged with Salmonella enteritidis. Br. Poult. Sci. 50:467478. doi:10.1080/00071660903110935

Mookiah, S., C.C. Sieo, K. Ramasamy, N. Abdullah, and Y.W. Ho. 2014. Effects of dietary prebiotics, probiotic and synbiotics on performance, caecal bacterial populations and caecal fermentation concentrations of broiler chickens. J. Sci. Food. Agric. 94:341-348. doi:10.1002/jsfa.6365

Niba, A., J. Beal, A. Kudi, and P. Brooks. 2009. Bacterial fermentation in the gastro-intestinal tract of non-ruminants: influence of fermented feeds and fermentable carbohydrates. Trop. Anim. Health. Prod. 41:1393-1407. doi:10.1007/s11250-0099327-6

Patel, S., R. Shukla, and A. Goyal. 2015. Probiotics in valorization of innate immunity across various animal models. J. Funct. Foods 14:549-561. doi:10.1016/j.jff.2015.02.022

Reid, G. 2016. Probiotics: definition, scope and mechanisms of action. Best Pract. Res. Clin. Gastroenterol. 30:17-25. doi:10.1016/j.bpg.2015.12.001

Setlow, P. 2006. Spores of Bacillus subtilis: their resistance to and killing by radiation, heat and chemicals. J. App. Microbiol. 101:514-525. doi:10.1111/j.1365-2672.2005.02736.x

Shim, Y., S. Ingale, J. Kim, K. Kim, D. Seo, S. Lee, B. Chae, and I. Kwon. 2012. A multi-microbe probiotic formulation processed at low and high drying temperatures: effects on growth performance, nutrient retention and caecal microbiology of broilers. Br. Poult. Sci. 53:482-490. doi:10.1080/00071668.2012.690508

Siepert, B., N. Reinhardt, S. Kreuzer, A. Bondzio, S. Twardziok, G. Brockmann, K. Nöckler, I. Szabó, P. Janczyk, R. Pieper, and K. Tedin. 2014. Enterococcus faecium NCIMB 10415 supplementation affects intestinal immune-associated gene expression in post-weaning piglets. Vet. Immunol. Immunopathol. 157:65-77. doi:10.1016/j.vetimm.2013.10.013 
Skokovic-Sunjic, D. 2016. Clinical guide to probiotic supplements available in Canada: indications, dosage forms and clinical evidence to date. 2016 ed. International Scientific Association for Probiotics and Prebiotics, Sacramento, CA, USA. http://4cau4jsaler1zglkq3wnmje1.wpengine.netdna-cdn.com/wp-content/uploads/2016/01/clincial-guide-canada. pdf (accessed May 4, 2018).

Smolinska, S., D. Groeger, and L. O’Mahony. 2017. Biology of the microbiome 1: Interactions with the host immune response. Gastroenterol. Clin. North Am. 46(1):19-35. doi:10.1016/j.gtc.2016.09.004

Surendran, N.M., M.A. Amalaradjou, and K.A Venkitanarayanan. 2017. Antivirulence properties of probiotics in combating microbial pathogenesis. Adv. App. Microbiol. 98:1-29. doi:10.1016/bs.aambs.2016.12.001

Uyeno, Y., S. Shigemori, and T. Shimosato. 2015. Effect of probiotics/prebiotics on cattle health and productivity. Microbes Environ. 30:126-132. doi:10.1264/jsme2.ME14176

Yeoman, C.J., and B.A. White. 2014. Gastrointestinal tract microbiota and probiotics in production animals. Annu. Rev. Anim. Biosci. 2:469-486. doi:10.1146/annurev-animal-022513-114149

Yirga, H. 2015. The use of probiotics in animal nutrition. J. Probiotics Health 3:132. doi:10.4172/2329-8901.1000132

Zhang, Z., and I. Kim. 2014. Effects of multistrain probiotics on growth performance, apparent ileal nutrient digestibility, blood characteristics, cecal microbial shedding, and excreta odor contents in broilers. Poult. Sci. 93:364-370. doi:10.3382/ ps.2013-03314

Zhang, L., L. Zhang, X. Zhan, X. Zeng, L. Zhou, G. Cao, A. Chen, and C. Yang. 2016. Effects of dietary supplementation of probiotic, Clostridium butyricum, on growth performance, immune response, intestinal barrier function, and digestive enzyme activity in broiler chickens challenged with Escherichia coli K88. J. Anim. Sci. Biotechnol. 7:3. doi:10.1186/ s40104-016-0061-4 